\title{
Finding by Site or System
}

National Cancer Institute

\section{Source}

National Cancer Institute. Finding by Site or System. NCI Thesaurus. Code C36278.

A term that refers to the classification of a clinical or laboratory finding according to the anatomic site or system that is involved. 